\title{
AC 2012-4303: OPEN PROCESS FOR ENTREPRENEURING TEAM COL- LABORATION: PARALLELS FROM AN ACADEMIC RESEARCH TEAM TO THE START UP THEY STUDIED
}

\section{Prof. Barbara A. Karanian, Stanford University}

Barbara A. Karanian, Ph.D. teaches graduate design methods and a new REVS class on the car experience in the College of Engineering at Stanford University, using applied psychology and art for storytelling to facilitate student progress from the idea and prototyping phases to delivery. With a focus on entrepreneurial leadership, Karanian makes productive partnerships with industry and forms collaborative teams from the areas of engineering, design, psychology, and communication. She was the Michael T. Anthony Professor at Wentworth Institute of Technology in Boston from 2005-2009. Karanian paints pictures.

Ms. Mona Eskandari, Stanford University

Akshit Aggarwal, Stanford University

Akshit Aggarwal is a graduate student at Stanford University, majoring in computational and mathematical engineering.

Felipe Pincheira, Stanford University

Ms. Rebecca Rose Krauthamer

Mr. Gregory Kress, Stanford University

Greg Kress is a Ph.D. student of mechanical wngineering in the Stanford Center for Design Research. His research activities include developing tools and methods to encourage radical creativity, modeling team cognition to predict design performance, and investigating trends in the future of the internet. Kress is an alumnus of the University of Michigan Engineering Physics program, the Co-founder of the Imaginary Lab at Stanford and a lecturer and coach for the capstone master's course ME310: Global Design Innovation. 
Open Process for Entrepreneuring Team Collaboration: Story Parallels from an Academic Design Team to the Studied Start-Up

Eskandari, Mona; Pincheira, Felipe E.; Krauthamer, Rebecca; Aggarwal, Akshit; Forouhar, Pamon; Dua, Janesha; Peng, Christine; Kress, Greg; Karanian, Barbara 


\begin{abstract}
Increasingly, student entrepreneurial ventures begin as emotional connections, artistic experiences, and expectations for delivering on research teams. This paper explores student team progress and responses to roadblocks while helping a maturing Silicon Valley start-up IMVU consider the role of avatars, creative expression and social interaction in the virtual world. Our goal was to seek a deep understanding of why users choose to spend their time and money to take part in the online community IMVU all the while creating a productive entrepreneurial team atmosphere. This paper explores the unique dynamics of the research team during the evolving investigation and delivery phase, all the while simultaneously examining the underlying emotions and motives of participants in one virtual community. We start with the belief that every new research team is like a start-up company and there are commonalities or differences between the student academic research environment and the company's organizational culture. Our collaborative research team includes members from areas of engineering, design, psychology, and communication. In this paper, we intend to correlate the factors that make the design team effective, utilize the findings to guide new student teams, and facilitate progress across the stages of the project. Two factors set the stage for insights on entrepreneuring: 1) evolving research team dynamics, and 2) the need-finding interactions with users both inside and outside the industry environment studied (IMVU). Surprising discoveries include a strong gender imbalance in the community as well as users reporting that online "was basically real life." A palette of stories abstractly parallels the student design team to the start-up they studied. Concepts include: self motivated, ambiguity readiness level, passion, and empathy. The team leader knowingly discussed using an open-process approach. Members noted a considerable lack of reluctance to prototype methods and team presentations; they also reported a deliberate lack of specific planning that they believe contributed to an entertaining and productive team ambiance. The full experiment offers stunning stories and compelling implications for creating effective design interventions in team-based engineering and design classes as well as for those pursuing the stories of compassion, empathy, and transformation in entrepreneuring.
\end{abstract}

Key words: Open-team process, Entrepeneuring, Design Thinking, IMVU, Social Participation, Empathy

\title{
1. Introduction and Overview of Design Team Dynamics
}

Our diverse group, composed of graduate mechanical engineering students, a psychology major, a communication major, and professors across departments of engineering design and communication, began a conversation about working as a research team that would focus on the design needs of a maturing start-up social community [1] for academic credit. Many have discussed the emotional dynamics of work groups $[2,3,4]$ and contrasted academic team norms to industry team norms [5]. The formative stages of a group are rather exciting; the unraveling of events sets a powerful precedence for the long-term team dynamics. During the first meeting, the 
student lead comfortably stated, "I am not going to set any strict guidelines, structures, or methods for our industry project together..." Such team dynamics set the stage for the thrill of anticipation and the provocative wonders of what would transpire.

The results of this particular class experiment and other industry driven design team research experiments have been documented $[6,7]$. Researchers have even extensively compared and discussed the design team process of students and professional engineers and/or designers [8]. Scholarship has contributed to our understanding of cultural factors and the regional advantages of Silicon Valley for breakthrough impact $[9,10]$. Furthermore, while extensive observations of formal small group learning of design teams in large classes and innovations in lectures have also been explored, it is unique to consider a knowingly utilized "open process" approach for the team; a process which by definition is open and available to alternate, change inputs, and add new perspective - all dependant on the team's decisions and dynamics (strikingly similar to that of a startup company). Our original intent for this paper was to consider the commonalities and differences of the academic team to the start-up company studied. We planned to explore the relevant factors through the reflective perspective of team members. We then intended to explore and elaborate on the similarities of the student team and transformative executive team culture. And of course, there were struggles. How does one examine a process we claim to advocate and want to implement? Our struggle is typical of the learning curve of enthusiasm and creative promise during the start-up stages of a team. First, we do not have a "control" group or research team for comparison yet, so we cannot comment on the effectiveness of this process over any other in data terminology. Second, we acknowledge that we have considered similarities - maybe analogous to a startup in an abstract sense - but have only limited and proprietary knowledge of the transformative depth of the levels of organizational culture [11, 12]. What we do know may not be written in this context.

Therefore, we have changed the original intent for this paper and will shift the approach to examine our assertions concerning the success of an "open-process" team. Two questions provide the preliminary focus: 1) how do we introduce the elements of the team "open process" approach? And, 2) what are ways to develop a method to study the concepts of "open process" to illuminate its effectiveness for delivering innovation?

Our primary motive is to understand and define why our start-up team "open process" approach was effective by considering the concepts and ways that the overall culture of the academicindustry environment accommodates this participation. 


\section{Examination of Design Team Dynamics}

In this paper, we consider the stories of a five person team and share discoveries that indicate a unique "open process" team development across three consecutive ten-week academic terms. The notion of "open process" for team success is unique, exciting, complex, amusing, and challenging for large team work and depends on academic culture, and organizational commitment as much as the emotional make-up of the individuals. This preliminary examination uses the stories of team process as one dimension for considering ways to thrive across the developmental stages of a project. We seek to break new ground in understanding design for academia-industry innovation, specifically in cross-discipline engineering design teams.

"Open process" for start-up team success refers to both the motivational and emotional make-up of the individuals on the team and the specific behavioral element they use to form connections for effective progress, within the culture of the environment. Group dynamics theory and organizational culture applications to the formative stages of teams and start-ups suggests that without new approaches, the tendency is to stay within the comfort zones of routine and resist pushing the boundaries to pursue novelties.

\subsection{Concepts}

Using a grounded theory approach, and drawing on applied psychology, design thinking, and leadership theory, our team prototyped four concepts with which to classify the social interaction and emotional behaviors of the "open process" team (see Table 1) [13]. Four concepts: selfmotivated; ambiguity readiness; passionate social connection; and empathy are considered. Each concept can be represented by a small spectrum between two extremes, enabling better resolution. Our goal is to explore the meaning of the concepts for impact on team dynamics, parallel to developmental stage and culture, to qualitatively determine the interaction, if any, between two or more of the concepts.

\section{Table 1: Concept Definitions}

$\begin{array}{ll}\text { Self-Motivated } & \text { Represents a range of behaviors that are characterized on a continuum } \\ \text { (MO) } & \text { with bipolar extremes: "ability to work for reasons that go beyond } \\ \text { time, money, or status" "self-driven interactions at one extreme, and } \\ \text { "external driven" interactions on the other [McClelland, 1964; 1987; } \\ \text { Goleman, 2004]. }\end{array}$

Ambiguity Readiness (AR)

Passionate Social Connection (SC)
Represents a person's ease with ambiguity along a developmental readiness spectrum for work, team path, and the lack of details and logistics for product delivery in and outside the environment [Stefik \& Stefik, 2006).

Linked to responses that express a sense of intrigue, interest, excitement, and a general reported vibe for staying in the moment of the work with real and tangible applications [Taylor \& Karanian, 2008]. 
Empathy (EMP)
Tracks the extent to which a student is personally capable of understanding the emotional make-up of other people, stepping into the shoes of the user and others (in terms of virtual communication, team communication, interactions at IMVU) and treating them accordingly [Goleman, 2004; Barry, 2007].

\subsection{Analysis}

Content for analysis for the overall concepts concern the emotional and motivational make-up for the individuals on the team and heavily draws from the research on effective leadership and emotional intelligence [13].

Self-motivated refers to the capacity to be visionary about personal achievements [14] and the "ability to work for reasons that go beyond time, money, or status" "self-driven interactions at one extreme, and "external driven" $[15,16]$ interactions on the other. This category seeks to answer how above and beyond an individual goes regardless of the task they are charged with.

Ambiguity Readiness represents a person's ease with ambiguity along a developmental readiness spectrum for work, team path, and the lack of details and logistics for product delivery in and outside the environment and draws from the theories of radical innovation [17] and has formative elements of design thinking principles [18]. A process is developmental over time and there exists no manual for maneuvering and planning the outcomes of a startup.

Passionate Social Awareness includes responses that express a sense of intrigue, interest, excitement, and an artful way of connecting [19,20]. Also evident is social ease and a capacity to refresh quickly, and for staying in the moment of the work with real and tangible applications for iterative work. A team relies on the cooperation of all the parts; it is essential that there is a constant feedback loop between team members.

Empathy tracks the extent to which a participant is personally capable of understanding the emotional make-up of other people, stepping into the shoes of another on the team and feel "with" vs. for another. It also refers to the foundation hallmark of design thinking in the context of understanding the user (in terms of virtual communication, team communication, interactions at IMVU) and treating, building, and designing for them accordingly [21]. Extensive expansion about the analysis is in progress. The empathy characteristic is critical when it comes to team function and interactions between team members; each teammate must be aware of the inputs and contributions of each person and ensure that people are felt valued and appreciated to improve team dynamics.

\subsection{Approach}

The procedure was considered a preliminary experiment, and builds on the previous parallel work in the Tell/Make/Engage (TME) methodology [22]. No single prototype consideration of this particular Tell/Make/Engage class approach existed before the current analysis. 
Three expectations were the foundation for the approach:

1. Group engagement can be fostered through an open process and storytelling.

2. Group engagement is observable as open process creates an unspoken knowing.

3. Group engagement contributes to shared vision and real progress for innovation.

\section{Stories}

Students perform and deliver their best work when they feel a sense of ownership over the task and are self-motivated to complete it (MO). Our team had no deadline or reward structure in place to motivate project work, and tasks were rarely delegated by the team lead. Rather, students were encouraged to make progress in whatever way they felt would be the most helpful, alone or in groups, which resulted in several spontaneous new directions emerging over the course of the project. Also, the potential for real impact in making tangible changes to the product provided a subtle motivation for project work and encouraged us to make our findings directly applicable and easily communicated. Students were chosen for the team because of the degree of self-motivation and interest that they expressed, and were encouraged to follow lines of inquiry that were of particular personal interest to them.

Particularly in the context of social research, it is important for researchers to maintain an open mind and measured approach in communicating with subjects, and not allow their personal beliefs, emotional reactions or prior impressions to cloud their interpretation of subject responses or influence the study. In this team, we needed to suspend our previously held beliefs and the persistent stereotypes about virtual communities and online social interaction. In a handful of circumstances, we encountered surprising, sometimes even shocking, behavior that challenged the research team in their ability to empathize with these users. We were able to successfully use an empathy-driven approach (EMP) to explain these behaviors from the perspective of real-world users. One team member in particular struggled with maintaining an impartial and non-judgmental tone in user interviews because she let her own reactions guide the conversation rather than empathizing with the feelings and reactions of users. This was a threat to the quality of the data, the stability of the team dynamic and potentially, even the wellbeing of our participants.

In another instance, a team member narrated how, before joining graduate school himself, he had approached the then-current graduate students at his new university to de-mystify the graduate school environment and aid him to depart from feeling overwhelmed and confused from it all (MO). The senior students helped him immensely, from understanding the culture of the place to selecting useful courses. Most importantly, they helped him understand the value of mentorship. Our team member was greatly moved by their helpful nature, and decided to pass on the gesture to future incoming students (EMP). Since then, he has made sincere efforts to empathize with new incoming students, stepping into their shoes $\&$ helping resolve the issues they face.

The implicit hierarchy in the team was downplayed in virtually all team contexts, especially when periodically presenting our findings to the client as a group. In these sessions, all group members spoke freely and equally and were allowed to (and did) disagree with each other and 
even the client in some instances. Decisions, reports, and presentations were prepared collaboratively such that any team member could have delivered any part of the presentation without hesitation. Maintaining a "flat" hierarchy allows for equal sharing of ideas and input, and students not feeling marginalized in a team. This forms a positive feedback loop with the atmosphere of self-motivation and empathy, encouraging team members to communicate their own ideas better and to be less dismissive of the ideas of others.

The open flow of ideas and communication established by the "open-team process" could be a double-edged sword if not managed properly. During one of the team discussions, the floor was opened to ideas for the final project. The "open-team process" utilized by the group sparked several creative ideas, often one feeding from each other. However, the lack of creative barriers and boundaries (AR) was an incentive for team members to come up with diverse ideas. In fact, team members spent multiple meetings talking about new ideas and did not come to a final decision, until prompted to work within the framework imposed by a anxious team member. While this one team member was not comfortable with ambiguity for ambiguity sake (AR) this story supports a relevant idea. Although, an open process is certainly beneficial, the creative process should be facilitated by a team member who can effectively guide the discussion and keep the team on track to achieve its goals within deadlines.

Still, the developmental progress and capacity for ambiguity (AR) of the individual team members and the overall team readiness for deciphering ambiguous product work proves crucial. Varying degrees of student readiness were evident. In one instance, a student participant claimed, "I don't know what we are doing anymore." Yet her questioning of the meaning, relevance, and her overall capacity to continue did not erode the team commitment. Instead, it offered an opportunity for team conversation and a new focus on how to approach the executive team of the company; such an event was a living example of the "open process" in action. Another student even reported that the lack of direction allowed the team members to learn each other's strengths and weaknesses before starting the work process. "The organic flow of ideas allowed a natural group organization to emerge (SC)," she noted. "It quickly became apparent who would be the 'big ideas' team members, who would be the member to organize those ideas, who would be the members to push us forward from the idea stage to implementation, etc." It became an unspoken sub-goal of the team to encourage open and respectful dialogue in favor of giving each member an informal opportunity to present themselves as well as their ideas. In line with the theory of contingency-based business planning, discussion for discussion's sake took precedence over planning [24].

An artful way of socially connecting and intrigue (SC) demonstrate a particular gift that the team exhibited on multiple occasions. One memorable moment occurred when the team led a presentation of findings to the 110 member company at a luncheon. While the presentation was scheduled for 20 minutes - the members of the company were so riveted by the truly connected team - and their connection to the company - that the meeting/presentation extended to 90 minutes. The frenzy of an industry pace tends to set more stringent time constraints on the possibility for further social interaction. Yet, everyone socially adjusted (SC). During a later, more formal presentation, the student team leader, with team participation, designed a trophy and presented it to the CEO; the result was a simple yet exquisite abstraction of the design findings and a metaphorical tribute to the entrepreneuring vision of the both the student team 
and the transformative executive team. Following the meeting, the student lead claimed to the executive team, "This is the most fun I have ever had working on a team."

\section{Insights from Stories}

Details for stories highlighting the elements for each concept and details on method development suggests three specific discoveries:

-An open process approach works well in a start-up team environment when a diverse group chemistry has a balancing blend of motivational (MO), ambiguity readiness (AR), social connection (SC), and empathy (EMP). The gender composition was predominantly female and may well have contributed to impressive developmental progress.

-An open process produces a powerful unspoken knowing that is an intangible but gripping active ingredient for shared vision, real progress, and actionable results.

-The entrepreneuring successes and innovative results of an open process team environment may outweigh the frustration and pain of disappointments along the way, or when projects fail.

When the developmental progress of the start-up team goes well, stunning successes appear to be connected to the right team chemistry along with the right balance of MO, AR, SC and EMP. Perhaps the idea that everyone on the team will take the time to listen, and seamlessly co-develop the design ingredients of start-up excitement parallel the virtual community they studied. The team gender composition also presented a parallel. Similar to the user and customer base at the company, the academic team was predominantly female. This set the stage for immediate empathy and an inclusive care for the possibilities of transformative design. During the team's formal presentation of findings to the executive officers at the company, the CEO made what was implicit in their work explicit. He encouraged, "As you go through the presentation might you help us understand the $2 / 3$ female user base from your experience, what exactly you felt, and how that influenced what you prototyped, and your recommendations."

The unspoken knowing during the planning and across the stages of their work produced actionable results. In another related case, the team rehearsed a featured short film depiction of their work, and made visible how their process was the foundation for success. The results exceeded the group's expectations. They trusted each member to fulfill their responsibilities and went beyond the comfort level of simple ambiguity during the building phases with little worry that it would not be delivered well and on time.

Failures and roadblocks occur. While this particular team did not suspend the development of their work, it is certainly likely that the open process approach will not prevent failure and the ultimate shutdown of a project. In the featured team case, there was a moment of disappointment due to an uncomfortable discovery that threatened project success. They discussed the confusion with each other, considered the implications for their work with their professor, and decided to both document what transpired and create the result as a design 
intervention and a key finding. Was this something new or was the company aware of what they found? Entrepeneuring on the team was evident as they questioned the possibility of the new in their work. They acknowledged that to each other and planned to test that fact during their presentation to the entrepreneurial company they studied. What if the team response had been different? If they considered the setback too difficult and the team did not have the emotional and behavioral capacity (MO, AR, SC, EMP) to continue, despite the surprise development, the outcome might have had the potential for disaster.

Exploring the "open-process" team and interpretations for success has dramatic similarities to the design thinking process. Applications to the human centered aspects of design thinking include existing and even thriving with ambiguity and indefinite design prompts, recognizing the surprise for finding something new in the lucky accident, and more important, the high degree and capacity for empathy. This particular team's predominantly female composition suggests implications for work in engineering education on the impactful role of women engineers engaging as students today and persisting as entrepreneurial leaders tomorrow [25].

Commonalities also exist in our preliminary work with the scholarship on radical innovation; and the contributions for the impact of our research on design teams in academia, established industry, and start-ups. Most relevant is completing work that will attract excellent students, faculty, and leaders for transformative work in engineering education.

\section{Introductory Discussion of Team Process for Entrepreneuring Parallels}

While this is a preliminary and exploratory examination, we do believe that deciphering stories of unique "open-process" teams insinuates something refreshingly original. Although it may be premature to commit to a new method and our methodology, we are in the process of deciphering the meaning, considering the challenges, and are assured of the developments of this term to prove worthwhile and facilitate another deeper look in another case.

Two concluding questions need to be addressed in next steps for entrepreneuring implications: 1) how do we introduce the elements of the team "open process" approach? And, 2) what are ways to develop a procedure to study the concepts of "open process" and illuminate its effectiveness for delivering innovation? Further work needs to test an open process approach in another prototype, and optimally in other environments.

It is helpful to understand what a fundamental shift it was for some of the students to learn how to participate, exist, and even thrive in an open process approach. Our preliminary case analysis suggests promising trends with implications for how and why new student teams designing for startups like IMVU become and remain extremely viable and likely to innovate. First insights suggest that introducing an open process approach works well when it is in an environment that promotes individual initiative and high-level responsibility for accomplishment and invention. The emotional and behavioral make-up, and start-up chemistry worked well in this case. But might a similar approach work in other academic or industry environments? 
Factors of evolving research, team dynamics, and the interactions inside and outside the team, with the company at the IMVU environment set the stage for insights entrepreneuring. It is most encouraging that our participative team work has been successful for industry collaboration yet it is of particular interest of why we were successful.

Related research and parallel results on motivation, stories reflecting inner experience that drives innovation $[22,23]$, and collaborative cross-discipline dynamics inform our work. Currently, we are conducting further analysis of our case experience (and expanding our reflections) that will explore the implications of our findings on "open team process" in classroom team dynamics for academia-start up industry collaboration, as well as interventions for design based engineering teams.

\section{References}

[1] IMVU Homepage. Web. 08 Mar. 2011. <http://www.imvu.com/>.

[2] Bion, R. Psychoanalysis and Group Behavior.The Electronic Bulletin of Psychoanalysis, 1949.

[3] Tajfel, H. "Social identity and intergroup behavior." Social Science Information Vol. 13 (1974) pp. 65-93.

[4] Smith, K. \& Berg. D. Paradoxes of Group Life. Jossey-Bass. 1987.

[5] Hackman, J.R. Leading Teams, Setting the Stage for Great Performances. HBS press, 2002.

[6] Kress, G., Getz-Kikuchi, R. Price, T. and Karanian, B.Social Participation in the Virtual Universe.National ASEE annual proceedings, Vancouver, BC, June, 2011.

[7] Smith, K. et al. Connecting and expanding the engineering education research and innovation special session. FIE, Oct. 14, 2011.

[8] Wankat, P; Felder, R. \& Smith, K.The scholarship of Teaching and Learning in Engineering. 2002.

[9] Saxenian, A. L. The New Argonauts: Regional Advantage in a Global Economy. Harvard University Press. 2006

[10] Stefik, M. \& Stefik, B. Breakthrough: Stories and Strategiesof RadicalInnovation: MIT press, 2004.

[11] Schein, E. Organizational Culture. Jossey-Bass, 1985.

[12] Heifetz, R. and Linsky, M. Leadership on the Line.Harvard Business School Press, 2002.

[13] Goleman, D. What Makes A Leader? Harvard Business Review, 2004. 
[14] Baum, J., Locke, E. \& Kirkpatrick, S. 1998.A longitudinal study of the relation of vision and vision communication to venture growth in entrepreneurial firms.Journal of Applied Psychology, 83. 43-54

[15] McClelland, D., Power: The Inner Experience. Van Nostrand: New York, 1964.

[16] McClelland, D., Human Motivation. Van Nostrand: New York, 1987.

[17\} Stefik, M. \& Stefik, B. 2004. Breakthrough: Stories and Strategiesof RadicalInnovation: MIT press.

[18] Leifer, L. 2005. Center for Design Research at Stanford University, Design Process Improvement-A Review of Current Practice. Clarkson, P. Eckert, C. (Eds). 522-526.

[19] Taylor, S. \& Karanian, B. Working Connection: The Relational Art of Leadership. Aesthesis. 2008.

[20] Karanian, B., Taylor, S. \& Skogstad, P. 2008. Engineer as entrepreneurial leader: an artistic balancing act. National ASEE Meeting and Proceedings, Pittsburgh, PA.

[21] Barry, M. Innovation as a Learning Process: Embedding Design Thinking. CA Mgt. Review, 2007

[22] Karanian, B., \& Kress, G. (2010). “Tell/Make/Engage: Actions for Innovation.” In Proceedings of the $40^{\text {th }}$ anniversary Frontiers in Education Conference (FIE), October, Washington, DC.

[23] Karanian, B., Kress, G., Sadler, J. (2009). "Telling Design Stories: the Result or the Entrepreneuring Investigation" in Proceedings of the 17th International Conference on Engineering Design (ICED), Stanford, 2009.

[24] Honig, Benson (2004). "Entrepreneurship Education: Toward a Model of ContingencyBased Business Planning." Academy of Management Learning \& Education. Vol. 3, No. 3, pp. 258-273.

[25] Ohland, M; Sheppard, S, Lichtenstein, G,; Eris, O.; and Chachra, D. (2008). "Persistence, Engagement and Migration in Engineering Programs. ” Journal of Engineering Education, July. 